\title{
Realizing the Translational Promise of Psychophysiological Research in ASD
}

\author{
J. C. McPartland • R. Bernier • M. South
}

Published online: 28 November 2014

(c) Springer Science+Business Media New York 2014

\section{Introduction}

This special issue of the Journal of Autism and Developmental Disorders focuses on the contributions of psychophysiological research to scientific understanding of autism spectrum disorder (ASD). The past decade has brought considerable advances in the application of psychophysiology for examining mechanisms of function and dysfunction in ASD. These insights span the range of behaviors affected, including social communication, restricted and repetitive behaviors, sensory processing, and cognition. Psychophysiological methods hold promise to offer key insights into autism by elucidating the biological underpinnings of behavior, by informing clinical understanding through more nuanced characterization of the phenotype, and by guiding intervention via describing treatment targets, predicting response to intervention, and measuring outcomes. The current issue reflects the breadth and depth of these approaches to ASD by presenting, in a single volume, a range manuscripts employing electrophysiology, electromyography, cardiac measures, magnetoencephalography, and electrodermal response. The studies described in this special issue address scientific

J. C. McPartland $(\bowtie)$

Yale Child Study Center, Yale University, 230 South Frontage

Road, New Haven, CT 06520, USA

e-mail: james.mcpartland@yale.edu

R. Bernier

Department of Psychiatry, University of Washington, Seattle, WA 98195, USA

M. South

Department of Psychology and Neuroscience Center, Brigham Young University, Provo, UT 84602, USA questions related to social perception, sensory attention, interpersonal responsivity, social ability, memory, language, and other areas, and the populations involved in this work range from infants to adults with ASD, typical development, and other neurodevelopmental disorders, such as tuberous sclerosis and ADHD. The adoption of and enthusiasm for these techniques by ASD researchers is evident in the overwhelming response to our call for papers. A competitive review process yielded 25 outstanding manuscripts, representing the state of the science in psychophysiological ASD research. We are grateful to the editorial staff at Springer for embracing this enthusiasm and kicking off 2015 with publication of this "super issue" that exceeds standard limits for special issues or sections.

Psychophysiology is established as a critical component of ASD research, informing early detection, yielding insight into markers of dysfunction across symptom domains, and illuminating response to treatment. In the first work to document predictors of diagnostic outcome in infants at-risk for ASD, Elsabbagh and colleagues used event-related potentials (ERPs) to detect neural responses to eye gaze at $6-10$ months of age that predicted subsequent ASD diagnosis at 36 months of age (Elsabbagh et al. 2012). Psychophysiological approaches have revealed markers of dysfunction across multiple domains related to autism. In terms of social behavior, McPartland and colleagues capitalized on the temporal resolution of electrophysiology to reveal delayed neural responses to faces in individuals with autism (McPartland et al. 2004). Roberts and colleagues shed light on identified delays in the M100 component in response to simple sensory information in individuals with autism, suggestive of a psychophysiological endophenotype for autism (Roberts et al. 2010). By examining skin conductance response, South and colleagues demonstrated an association between autonomic 
response to a simple discrimination conditioning task and autism symptom severity, informing the neural functioning of structures implicated in fear conditioning in autism (South et al. 2011). Analysis of spectral EEG power has helped explain dysfunction in action execution/observation in autism and elucidate the relationship between neural functioning and imitative ability (Bernier et al. 2007, 2013). Finally, the application of electrophysiology to autism intervention has provided initial evidence of brain changes in response to behavioral intervention, highlighting the efficacy of treatments based on behavior analytic principles to specifically target and improve the efficiency of brain mechanisms involved in social behavior (Dawson et al. 2012). These and the many other innovative applications of psychophysiology to autism research highlight the significant contributions and essential role of these approaches to informing our understanding of ASD. This special issue highlights the wide application and utility of these methodologies for creating innovative, insightful, and practically informative science.

\section{Innovation, Insight, and Methodological Advancement: The Special Issue}

Building from these discoveries of the past decade, this volume offers novel empirical contributions, synthesizes existing work through comprehensive reviews, and provides critical guidelines for methodologically rigorous electrophysiological research in autism. The innovative studies reported in this issue span multiple constructs, populations, and approaches, all elucidating mechanisms and psychophysiological processes pertaining to ASD. This includes studies measuring basic brain organization during rest or very simple tasks and potential links to behavioral manifestations of ASD. For example, Maxwell and colleagues found significant associations between gamma power measured across the resting brain and behavioral reports of social function, while Machado and colleagues' quantitative EEG (QEEG) analyses inform understanding of repetitive behaviors. Gabard-Durnam and colleagues suggest the potential of frontal alpha asymmetry as an endophenotype for ASD even in very young infants, while Ghanbari and colleagues report MEG measurements of signal complexity compared to analyses of functional connectivity to link findings across neuroimaging modalities. Bink and colleagues report the utility of alternate psychophysiological measures for understanding ASD visà-vis comorbidities; in their paper, cardiac reactivity served as an indicator of both baseline and task-related effects of stimulant medication in ASD and ADHD. Schaaf and colleagues also used cardiac measures for the purpose of characterizing a disorganized parasympathetic response to a sensory challenge procedure. Stamoulis examined EEG rhythms to characterize potential neurodevelopmental delay in children born with tuberous sclerosis complex. Oberman and colleagues reviewed the potential of transcranial magnetic stimulation (TMS) in ASD research, concluding that careful, controlled clinical trials are necessary before TMS can be established as an effective intervention for ASD.

As reflected in the recently revised diagnostic criteria for ASD, there is increasing recognition of the critical contribution of fundamental sensory processing differences to autistic symptomatology. This emerging field is reflected across a range of techniques reported in this issue. An ERP auditory oddball paradigm reported by Donkers and colleagues suggests attenuation in young children with ASD for both early sensory response and later auditory attention mechanisms. Edgar and colleagues completed a MEG study with a large sample of children with ASD and showed atypical auditory processing compared to controls in the superior temporal gyrus during exposure to tones of various frequencies; atypical response was associated with language performance. A visual oddball paradigm reported by Westerfield and colleagues showed atypical ERPs in ASD reflect variability in attention. Two studies of interhemispheric transfer, employing different methods and developmental ranges, report contradictory conclusions. A sample of 14 school-aged boys with ASD in Lazarev and colleagues' study demonstrated reduced interhemispheric coherence during intermittent photic stimulation. In contrast, Clawson's research indicated no group differences in interhemispheric transfer time in a sample of older children and adolescents with ASD. These studies highlight the need for careful attention to measurement issues (including method, task development, and sample characteristics), yet they may also reflect the heterogeneity and potential developmental effects of ASD.

Psychophysiological techniques also inform understanding of higher-order learning in ASD. This issue includes reports linking response monitoring (in the errorrelated negativity) to severity of autism symptoms, especially to performance on theory of mind tasks. ReebSutherland and colleagues highlight the potential utility of eye-blink conditioning as a specific biomarker for ASD relative to other neurodevelopmental conditions. Massand and Bowler suggest that ERP markers of limited differentiation between semantic and episodic memory judgments may underlie episodic memory impairment in ASD.

Finally, psychophysiological experiments provide important insight into the nature of hallmark social difficulties in ASD. Coffman and colleagues reported on the first wellcontrolled study of sex differences in social perception in ASD, revealing clinically relevant discrepancies in the manner in which males and females with ASD process faces. 
Key and colleagues studied infant siblings of children with ASD and found that risk status was most saliently reflected in neural response to positive affect in the context of subtle facial expressions. The facial mimicry study reported by Deschamps and colleagues shows reduced sensitivity specifically to fear signals that may impair social reciprocity, while Smeekens demonstrated the utility of both autonomic and endocrine measures of stress response to studying reactivity in naturalistic situations. Three studies examined social interactions between children affected by ASD and their caregivers. Elsabbagh and colleagues showed that differences in ERP response to eye gaze in high- and normal-risk infants is more dependent on child characteristics in the highrisk group and on parent characteristics in the normal-risk group. Robeldillo and Albiol documented the effects of chronic stress on electrodermal response of caregivers for individuals with ASD. An innovative study of treatmentrelated changes in EEG by Van Hecke and colleagues showed that gamma band asymmetry reversed lateralization from right to left hemisphere following social skills intervention, and this change was associated with decreased social isolation and overall autism symptomatology.

This issue also includes several manuscripts that synthesize existing information rather than reporting on empirical investigations. Three comprehensive review articles capture and explore the relevant literature in the field of ASD and developmental disorders. These reviews span three key physiological domains: cardiac autonomic measurement (Benevides and Lane), eye-blink conditioning (Reeb-Sutherland et al.), and transcranial magnetic stimulation (Oberman et al.). Perhaps the most valuable contribution of this volume is the publication of the first consensus statement regarding best practices for electrophysiological brain recording in ASD. Despite the wide application of EEG and ERP methods in ASD, the field has lacked guidelines for uniform acquisition, processing, and analysis of these data. Increased systematization of controlled variables, such as EEG and ERP data acquisition, decreases variability across studies. Synthesizing discussion among field leaders at the International Meeting for Autism Research Special Interest Group for EEG/MEG, Webb and colleagues describe in detail a best practices approach for the electrophysiological study of autism by tailoring the classic guidelines for electrophysiological brain research described by Picton et al. (2000). This manuscript encompasses the collective practical knowledge of EEG scientists in ASD to set standards for even more effective research in the future.

\section{Unique Benefits of Psychophysiology Research in ASD}

The work published in this volume, along with the foundation of psychophysiological research on which it builds, highlights the unique strengths of these methods and their significant translational applicability. Expanding on Banaschewski and Brandeis' (2007) seminal review of electrophysiology's benefits for psychiatry, we highlight several unique strengths of psychophysiological research for studying neurodevelopmental disorders.

Psychophysiological methods are unique in their ease of use and appropriateness for an exceptionally wide range of developmental and functional levels. Most of these methods require only the tolerance of sensors on skin. They are highly movement tolerant, such that movement artifact is specific to a given trial and as such can be removed or avoided, retaining the integrity of a data recording session. The ease of use makes psychophysiological methods widely applicable for individuals who are minimally verbal or intellectually disabled, as well as for infants, as demonstrated by several of the manuscripts in this issue. Additionally, the simplicity of psychophysiological research methods renders them utilizable across levels of experience. This is reflected in the authorship of manuscripts in this special issue, which ranges from senior leaders in our field to graduate students.

A second strength of the application of psychophysiological measures to the study of ASD concerns the unique nature of the information that is obtained. In contrast to other imaging methods requiring measurement of blood flow, these methods directly record central or peripheral nervous system activity as it happens and at the pace at which it happens. While hemodynamic imaging methods capture a single data point across seconds, the methods applied here regularly capture 1,000 data points per second. Like other methods, these psychophysiological recordings enable assessment of signal strength; however, unlike other methods, they offer access to qualitatively distinct types of information. In other words, these approaches offer insight into the efficiency of psychophysiological functions across domains of information. For example, the degree of temporal sensitivity enables scientists to ascertain whether certain kinds of information are processed more efficiently than others. This is highly relevant for understanding developmental differences; ERP studies have provided key indicators of neural specialization with development, with the observation that the hallmark index of face processing in adults (the N170 component) begins as a slower precursor component in infants (the N290 component) that follows a protracted maturational course through adolescence (Taylor et al. 2004). In the particular case of ASD research, this temporal sensitivity supports investigation of functional differences in ASD that may be attributable to anomalies in processing efficiency rather than simply magnitude of activity. For example, individuals with ASD demonstrate decreased processing efficiency for social stimuli but comparable efficiency for non-social information (McPartland et al. 2011). 
A second benefit of the temporal precision of many psychophysiological methods is the isolation of distinct processing stages within a complex cognitive or perceptual process. In contrast, other neuroimaging methods condense information over time, effectively blurring distinct processes if they occur within the same region of cortex in a brief period of time. An analogy would be to that of a camera that has only a two-second shutter speed. A picture taken with such a camera would accurately and precisely reflect the captured subject only if one single event transpired in that place during those two seconds; this is unlikely the case when measuring biological processes. For example, human perception of a dynamic face relies on a rapidly occurring series of functionally distinct processing stages evident in the ERP: lowlevel visual perception at $100 \mathrm{~ms}$, face structural encoding at $170 \mathrm{~ms}$, higher-order face decoding (e.g., emotion, identity) at $250 \mathrm{~ms}$, and activity in the action-perception system across this time span. This level of precision affords a more sophisticated and nuanced analysis of preserved and intact function across specific facets of a functional domain. In ASD research, it is critically important to understand the neural underpinnings of symptomatology from this kind of mechanistic perspective.

\section{Translational Value}

The strengths of psychophysiological approaches lead to direct translational value. Key goals in the field of autism research include detecting atypical development earlier in life, empirically defining subgroups, measuring treatment outcomes, specifying mechanisms to target during treatment, and predicting treatment response. Psychophysiological approaches can realize key translational goals through their economy, scalability, sensitivity, and objectivity.

Many psychophysiological methods are inexpensive compared to other assays of biological processes. The costs associated with many neuroscientific methods may limit their practicality, but costs associated with psychophysiological methods are minimal once an acquisition system is in place. For example, upkeep costs for an EEG system consist of salt water, latex gloves, and basic maintenance of electrode nets. Additionally, many of these methods, such as EEG, are already located in health care facilities around the world, supporting feasibility of large-scale translation; in fact, EEG is already at the population level for auditory screenings of newborns in the United States. The low cost of psychophysiological methods and their widespread availability mean that, practically, applications of scientific discoveries for clinical purposes at a large scale can be realistic.

Psychophysiological approaches offer increased sensitivity relative to behavioral assessment. This allows for the identification of subtle activity patterns that may never be reflected at the behavioral level or that may not have developmentally emerged in behavior. Given that behavior is an amalgam of myriad neural processes, many of which are likely convergent or compensatory processes in the individual, some differences important to understanding ASD may only be evident at the reduced level of neural functioning. For ASD, a diagnosis based predominantly on behaviors that do not typically emerge until the second year of life or later, this sensitivity offers great promise for detecting atypical development prior to the emergence of reliably detectable behavior. This notion is evident in the use of psychophysiological methods in this special issue to detect atypical social development not yet evident in behavior.

A final strength of these approaches concerns their objectivity. These measures allow for perfect consistency even when compared to rigorous behavioral methods. A relevant example concerns diagnostic tools of autism. Through careful training, regular monitoring, and consistent readjustment, two expert clinicians can reliably achieve similar results when applying the same behavioral tool with a child with autism. Although the resultant codes will be highly concordant, they are unlikely to be exactly the same. In contrast, with comparable hardware set-ups and experimental batteries, identical information can be collected in separate locations. As our field moves increasingly toward multisite research studies, the ability to collect standardized and reliable data without cumbersome and crude clinical reliability procedures will render psychophysiological approaches a key advantage for clinical trials research.

\section{Moving Towards a Neuropsychology of Neuroscience}

Much of neuroscience aims to take a single marker, such as activation in a particular region of the cortex, and classify differential activation across groups as a marker of disease state, risk status, or treatment response. Given the complexity of ASD, this is unlikely to reflect the reality of the disorder. As both practicing clinical psychologists and psychophysiological researchers, we recognize substantial benefits of applying the concepts of clinical neuropsychology to neuroscience research. Rather than examining a single metric of performance, neuropsychologists measure a broad array of abilities potentially relevant to understanding function and dysfunction. With this breadth of related but distinct information, individualized profiles of ability and disability are derived to (a) understand categories of dysfunction in a more nuanced way and (b) apply treatment in a more individualized fashion. In this way, psychophysiological methods in particular hold the 
potential to deliver a similarly nuanced and multifaceted approach to neuroscience. The temporal sensitivity of this methodology allows for discrimination of distinct processes over a short span of time. These processes can inform individualized profiles of mechanisms of ability and disability in a given person. They can then be related to behaviorally measured functional correlates (as measured in the lab/clinic or via clinician or caregiver report in reallife settings). As shown in Fig. 1, brain data acquired in a short span of time (e.g., a $500 \mathrm{~ms}$ display of a moving face) can be parsed to characterize distinct processing stages, each relevant to function and dysfunction in ASD. This type of nuanced profile has translational promise for individualized intervention. That is, a person with a profile highlighting sensory dysfunction would likely benefit from different treatments than one showing delayed processing of emotional expressions. An individualized profile enables measurement of treatment response in terms of objectively, reliably-assessed change in a specific targeted domain. Profiles can also advance development of treatments that target focal mechanisms rather than behavioral symptoms, which may represent diverse component processes.

The identification of individualized profiles based on psychophysiological processes may serve the objective of developing empirically defined subgroups. This could provide opportunities for developing novel diagnostic taxonomies based on biological processes rather than descriptions of overt behavior (Cuthbert and Insel 2013). Most studies of ASD compare groups of individuals to control groups, with attempts at subgrouping the samples based on cognitive ability or some other descriptive behavioral measurement. The power of a scientific study to detect group differences is only as strong as the accuracy and sensitivity of the constructs applied to define groups. With the application of the methods described in this special issue, subtler and more accurate distinctions may help to advance the field. Importantly, profiles derived from psychophysiological measures may serve as the necessary correlate for genetic analyses, given the mechanistic proximity of neural functioning to genotype relative

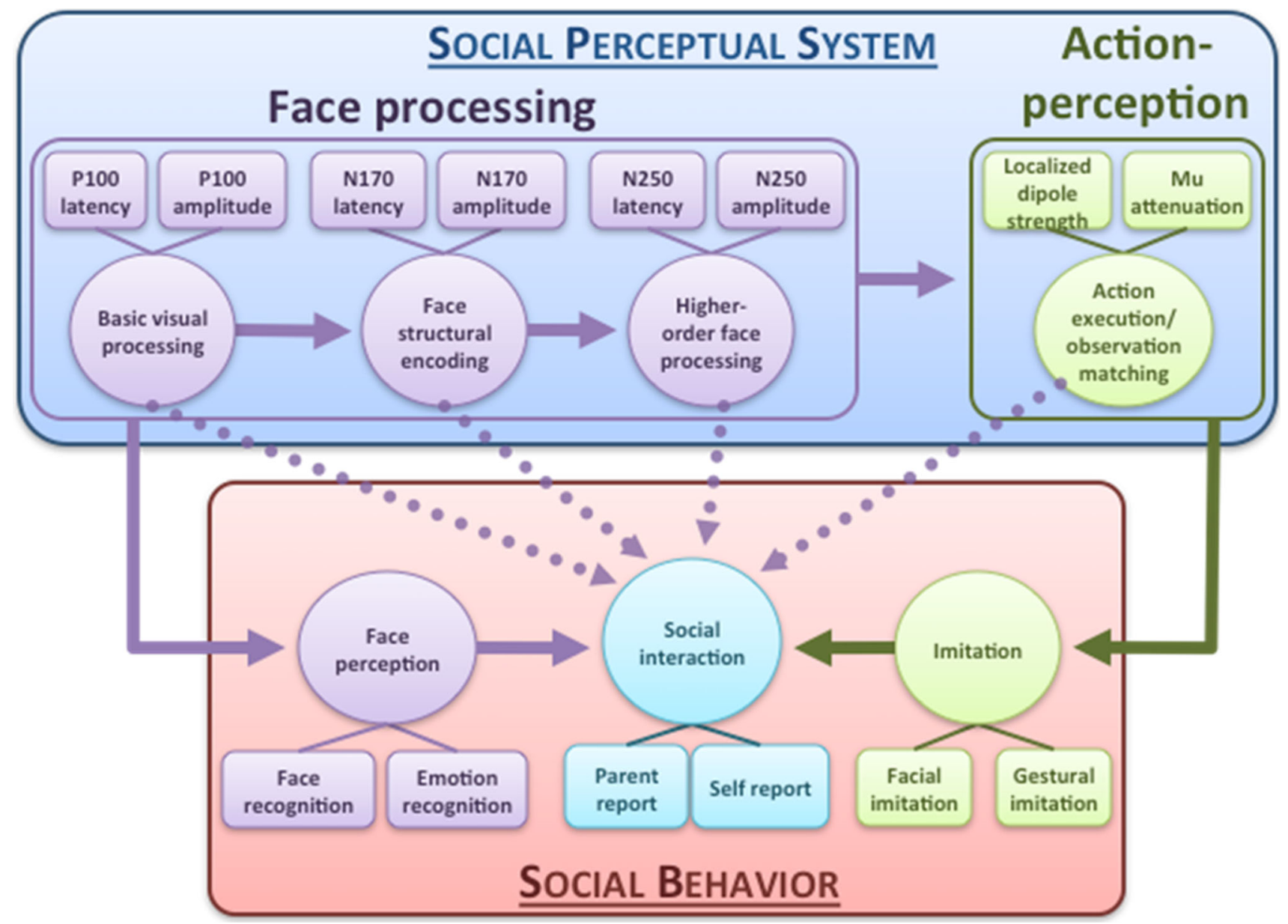

Fig. 1 Hypothesized model of relationships among social perceptual systems and social behavior in ASD. Dotted lines indicate potential alternate pathways of influence 
to complex behavioral symptomatology. As such, applying psychophysiology and working backwards from phenotype to genotype may contribute to advances in understanding the causes of autism. The application of this approach to identifying etiologically-defined subtypes has already proven informative in genetics-first studies and aided in parsing the heterogeneity of autism (Bernier et al. 2014). Psychophysiological assessment is poised to play a key role in this next step of autism research.

In conclusion, this special issue of Journal of Autism and Developmental Disorders highlights innovative studies that are advancing our understanding of ASD through psychophysiological research. Building upon the significant contributions these approaches have yielded in the past decades of autism research, the current manuscripts underscore the widespread applicability and translational utility of these approaches and provide compelling evidence that psychophysiological methods will continue advancing the field's understanding of autism for the welfare of the individuals and families affected by ASD and society at large.

Acknowledgments This work was supported by National Institute of Mental Health R01 MH100173 (JM), R01 MH100173-02S1 (JM), CTSA Grant Number UL1 RR024139 (JM), and R01MH100047 (RB). Its contents are solely the responsibility of the authors and do not necessarily represent the official view of the NIH.

\section{References}

Banaschewski, T., \& Brandeis, D. (2007). Annotation: What electrical brain activity tells us about brain function that other techniques cannot tell us-a child psychiatric perspective. Journal of Child Psychology and Psychiatry, 48(5), 415-435.

Bernier, R., Aaronson, B., \& McPartland, J. (2013). The role of imitation in the observed heterogeneity in EEG mu rhythm in autism and typical development. Brain and Cognition, 82(1), $69-75$.

Bernier, R., Dawson, G., Webb, S., \& Murias, M. (2007). EEG mu rhythm and imitation impairments in individuals with autism spectrum disorder. Brain and Cognition, 64(3), 228-237.

Bernier, R., Golzio, C., Xiong, B., Stessman, H. A., Coe, B. P., Penn, O., et al. (2014). Disruptive CHD8 mutations define a subtype of autism early in development. Cell, 158(2), 263-276.

Cuthbert, B. N., \& Insel, T. R. (2013). Toward the future of psychiatric diagnosis: The seven pillars of RDoC. BMC Medicine, 11, 126.

Dawson, G., Jones, E. J., Merkle, K., Venema, K., Lowy, R., Faja, S., et al. (2012). Early behavioral intervention is associated with normalized brain activity in young children with autism. Journal of the American Academy of Child and Adolescent Psychiatry, 5l(11), 1150-1159.

Elsabbagh, M., Mercure, E., Hudry, K., Chandler, S., Pasco, G., Charman, T., et al. (2012). Infant neural sensitivity to dynamic eye gaze is associated with later emerging autism. Current Biology, 22(4), 338-342.

McPartland, J., Dawson, G., Webb, S. J., Panagiotides, H., \& Carver, L. J. (2004). Event-related brain potentials reveal anomalies in temporal processing of faces in autism spectrum disorder. Jounal of Child Psychology and Psychiatry, 45(7), 1235-1245.

McPartland, J. C., Wu, J., Bailey, C. A., Mayes, L. C., Schultz, R. T., \& Klin, A. (2011). Atypical neural specialization for social percepts in autism spectrum disorder. Social Neuroscience, 6(5-6), 436-451.

Picton, T. W., Bentin, S., Berg, P., Donchin, E., Hillyard, S. A., Johnson, R, Jr, et al. (2000). Guidelines for using human eventrelated potentials to study cognition: Recording standards and publication criteria. Psychophysiology, 37(2), 127-152.

Roberts, T. P., Khan, S. Y., Rey, M., Monroe, J. F., Cannon, K., Blaskey, L., et al. (2010). MEG detection of delayed auditory evoked responses in autism spectrum disorders: Towards an imaging biomarker for autism. Autism Research, 3(1), 8-18.

South, M., Larson, M. J., White, S. E., Dana, J., \& Crowley, M. J. (2011). Better fear conditioning is associated with reduced symptom severity in autism spectrum disorders. Autism Research, 4(6), 412-421.

Taylor, M. J., Batty, M., \& Itier, R. J. (2004). The faces of development: A review of early face processing over childhood. Journal of Cognitive Neuroscience, 16(8), 1426-1442. 